\title{
Multiple Primary Lung Cancer Comprised of Adenocarcinoma and Adenoid Cystic Carcinoma: A Case Report
}

\section{jieyu xu}

Zunyi Medical College Affiliated Hospital: Affiliated Hospital of Zunyi Medical College https://orcid.org/0000-0003-0631-4302

Xiaorong Yang ( $\nabla$ yangxiaorong2003@126.com )

Affiliated Hospital of Zunyi Medical University

\section{Case Report}

Keywords: multiple primary lung cancer, adenocarcinoma, adenoid cystic carcinoma, immunohistochemistry, histopathology, case report

Posted Date: May 14th, 2021

DOI: https://doi.org/10.21203/rs.3.rs-506312/v1

License: (c) (i) This work is licensed under a Creative Commons Attribution 4.0 International License. Read Full License

Version of Record: A version of this preprint was published at Translational Cancer Research on March 1st, 2022. See the published version at https://doi.org/10.21037/tcr-22-166. 


\section{Abstract}

Background: Multiple primary lung cancer is a rare type of tumor, which is necessary to differentiate from a metastatic tumor.

Case presentation: We report a 67-year-old female with coughing and expectoration in the past eight days. Chest computed tomography revealed that there are two nodules in the patient's lung. Radiographic findings cannot distinguish between the two nodules and distinguish between primary and metastatic lesions. The patient underwent bronchoscopic biopsy and percutaneous lung puncture. Combined with morphological and immunohistochemical results, we concluded that this is a case of multiple primary lung cancers, consisting of adenocarcinoma and adenoid cystic carcinoma. In clinical practice, surgery is considered to be the first choice for the treatment of cases of multiple primary lung cancer. The patient received symptomatic treatment because of the metastases.

Conclusions: The article aims to report a rare case and emphasize the role of immunohistochemistry in diagnosing multiple lung cancer.

\section{Background:}

Multiple primary lung cancer (MPLC) is defined as the simultaneous or sequential occurrence of two or more primary malignant tumors in the same patient's lung. In 1932, Warren and Gates proposed the concept of multiple primary lung cancer. In MPLC, each tumor should have a definite manifestation, there should be differences between tumors, and the possibility of metastasis across lesions should be ruled out [1]. The incidence of MPLC is very low. We report a case of bilateral primary lung cancer characterized by adenocarcinoma and adenoid cystic carcinoma (ACC). In the present case, both the location (lower lobe of the right lung and the left main bronchus) and the combination (adenocarcinoma and adenoid cystic carcinoma) are uncommon.

\section{Case Presentation:}

A 67-year-old female patient was referred to our hospital for complaining of coughing and expectoration in the past eight days. She did not experience hemoptysis, fever, chest pain, dyspnea, or weight loss. She does not smoke and has no chronic diseases. Subsequent physical examination revealed thick breathing sounds over both lungs. On bronchoscopy, a popcorn-like pedunculated mass was found at the opening of the left bronchus. Chest computed tomography showed a small nodule at the beginning of the left main bronchus and a mass under the pleura in the posterior basal segment of the right lower lobe. The mediastinal lymph nodes were increased with some calcification.

Microscopically, the small gray-red nodule at the main bronchus was composed of glandular epithelial cells and myoepithelial cells. Tumor cells were haphazardly arranged in the shape of the ethmoidal foramen (Figure 1). Immunohistochemical analysis revealed that the glandular epithelium was positive for CK8/18, CAM5.2, and CK7 (Figure 2), while the basal cells were positive for P63 (Figure 3), P40, 
CK5/6, S-100, and CD117. On microscopy, the right lung mass consisted of tumor cells with irregular glandular arrangement (Figure 4). Most tumor cells grew and infiltrated the stroma. Immunohistochemical staining of tumor tissue demonstrated positivity for CK7, Napsin-A, and TTF-1 (Figure 5). The above results suggested that the tumor origins of the two lesions were different. After consultation, we diagnosed it as MPLC.

In addition, on chest CT, a metastatic lesion was shown in the sixth anterior rib on the left. The patient received symptomatic treatment including anti-infection, bronchodilation and nutritional support since surgery was not indicated. A gene drive test for lung cancer will be conducted during the upcoming follow-up, and targeted chemotherapy drugs will be selected according to the results.

\section{Discussion And Conclusions:}

Multiple primary lung cancer is rare, with a reported incidence of $3.7 \%$ to $8.0 \%$. This number has increased due to the development of clinical diagnostic techniques [2]. Like single lung cancer, MPLC occurs at an average age of 63.4 years, and $36.7 \%$ of patients are female [3]. It usually occurs unilaterally [4]. In this case, CT demonstrated that the lesions occurred in both lobes of the lungs.

It is repored that squamous cell carcinoma plus squamous cell carcinoma is the most common type $[5,6]$. The present case is composed of an adenocarcinoma and adenoid cystic carcinoma, which are rarely reported. Adenoid cystic carcinoma of the lung is a rare type of tumor that makes up $0.04-0.2 \%$ of primary pulmonary tumors[7]. It more commonly involves the salivary glands in the head and neck [8]. Histologically, this tumor exhibits three main growth patterns: the cribriform, tubular, and solid patterns. ACC of the lung is characterized by its expression of myoepithelial markers, which include P63, SMA, Ckit, and S-100 protein. Once pulmonary ACC is identified, it becomes essential to determine whether the lesion represents distant metastasis or primary lung cancer [9]. In order to rule out the possibility of metastatic diseases, we conducted a general otorhinolaryngological examination to try to seek a primary salivary gland tumor. It turned out that there is no abnormalities in the ears, nose and throat.

Furthermore, it is also critical and challenging to distinguish whether multiple lung cancers are independent or related. That is, to differentiate multiple primary cancers from intrapulmonary metastases. The former is typically treated surgically, and the prognosis is good. However, the latter involves systemic chemotherapy, and the prognosis is poor [10]. The American Joint Commission on Cancer has adopted comprehensive histologic assessment (CHA) as a diagnostic criterion [11]. The proposal of CHA provided a new basis for differential diagnosis. The contents of CHA include the percentage of various histologic subtypes, nuclear features, and stromal characteristics. This is particularly useful while evaluating multiple lesions that exhibit the same tissue type. The principle of immunohistochemistry technology is that antigen and antibody can combine specifically, so as to characterize and locate the specific antigen or antibody in tissues and cells. It can be used as a first-line method for differentiation and diagnosis in cases where imaging cannot distinguish between two tumor types. In addition, in clinical practice, the integration of radiology, histopathology, and integrated genomic 
features by multidisciplinary teams promotes a more accurate diagnosis of MPLC [12]. Thus, to further identify whether it was a primary tumor or metastatic cancer, we used histopathology to identify the two lesions from the source. As in this case, the nodule in the left main bronchus expresses both basal cells and glandular epithelial markers, leading to the diagnosis of adenoid cystic carcinoma of origin. The right lung mass expresses TTF- 1 and NapsinA, which we confidently diagnosed as originating from adenocarcinoma.

Unlike intrapulmonary metastasis, MPLC often presents as an early-stage disease [13]; thus, surgical treatment should be considered as the modality of choice for managing MPLC patients [14]. To date, radical surgery is the primary therapy for MPLC. However, the optimal extent of surgical intervention has not yet been standardized [12]. In Tie's article, he put forward anatomical resection, which is defined as lobectomy, double lobectomy, and pneumonectomy plus lymph node dissection, with adequate pulmonary reserve. When the patient has limited lung function, lobectomy with sublobectomy or sublobectomy alone is also possible. Among these, anatomic segmental resection is preferred for sublobar resection[4].Unfortunately, due to the distant metastases in our patient, we had to treat her conservatively. For the early patients who are medically inoperable in clinical, experts have proposed the treatment method of stereotactic body radiotherapy (SBRT). Its effectiveness and security have been proved [12]. However, the efficacy of SBRT for patients in advanced stage needs further research.

\section{Abbreviations:}

1. MPLC: Multiple primary lung cancer

2. ACC: adenoid cystic carcinoma

3. CHA: comprehensive histologic assessment

4. SBRT: stereotactic body radiotherapy

\section{Declarations:}

\section{Ethics approval and consent to participate}

Not applicable.

2. Consent for publication

Written informed consents were obtained from the patients or family of the patient for publication of this Case series and any accompanying images.

\section{Availability of data and materials}

The datasets used and/or analysed during the current study are available from the corresponding author on reasonable request. 


\section{Competing interests}

The authors declare that they have no competing interests.

\section{Funding}

None.

\section{Authors' contributions}

XRY made substantial contributions to acquisition, analysis and interpretation of patients' data and revised the manuscript. JYX interpreted the data and drafted the manuscript. All authors read and approved the final manuscript.

\section{Acknowledgements}

None.

\section{References:}

1. Warren S, Gates O. Multiple primary malignant tumors: a surgery of the literature and a statistical study. Am J Cancer. 1932;16:1358-414.

2. Jia XL, Zhang LP, Wu W, Zhang W, Wu G. Driver mutation analysis and PD-L1 expression in synchronous double primary lung cancer. Appl Immunohistochem Mol Morphol. 2018;26:246-53.

3. Tie H, Luo J, Shi R, Li Z, Chen D, Wu Q. Characteristics and prognosis of synchronous multiple primary lung cancer after surgical treatment: a systematic review and meta-analysis of current evidence. Cancer Med. 2021;10:507-20.

4. Liu XD, Qu Y, Lu SS. Synchronous double primary lung cancer: a report of three cases. Chin J Cancer Res. 2014;26:17-21.

5. Rohwedder JJ, Weatherbee L. Multiple primary bronchogenic carcinoma with a review of the literature. Am Rev Respir Dis. 1974;109:435-45.

6. Ferguson MK, DeMeester TR, DeLauriers J, Little AG, Piraux M, Golomb H. Diagnosis and management of synchronous lung cancer. J Thorac Cardiovasc Sur. 1985;89:378-85.

7. Krifa M, Bdioui A, Lajmi Z, Missaoui N, Hmissa S, Mokni M. Primary adenoid cystic carcinoma of the lung: a case report and literature review. Heliyon. 2021;7:e06206.

8. Sharma VJ, Gupta A, Yaftian N, Ball D, Brown R, Barnett S, Antippa P. Low recurrence of lung adenoid cystic carcinoma with radiotherapy and resection. ANZ J Surg. 2019;89(9):1051-5.

9. Suzuki M, Koyama R, Uchida Y, Ogushi K, Otake S, Yamamoto H, Mogi A, Shirabe K. Primary adenoid cystic carcinoma of the lung in 14-year-old boy. J Pediatr Surg Case Rep. 2020;56:101432.

10. Hitomi Kawai K, Iguchi N, Takayashiki, Okauchi S, Satoh H. Metachronous isolated contralateral lung metastasis from pulmonary adenosquamous carcinoma with EGFR mutation. Acta Medica (Hradec 
Kralove). 2020;63:141-4.

11. Rami-Porta RAH, Travis WD, Rusch VW. AJCC Cancer Staging Manual. 8 ed. France: Springer International Publishing; 2017.

12. Zhao L, Liu C, Xie G, Wu F, Hu C. Multiple primary lung cancers: a new challenge in the era of precision medicine. Cancer Manag Res. 2020;12:10361-74.

13. Jiang L, He J, Shi X, Shen J, Liang W, Yang C, He J. Prognosis of synchronous and metachronous multiple primary lung cancers: systematic review and meta-analysis. Lung Cancer. 2015;87:303-10.

14. Yang H, Sun Y, Yao F, Yu K, Gu H, Han B, Zhao H. Surgical therapy for bilateral multiple primary lung cancer. Ann Thorac Surg. 2016;101:1145-52.

\section{Figures}

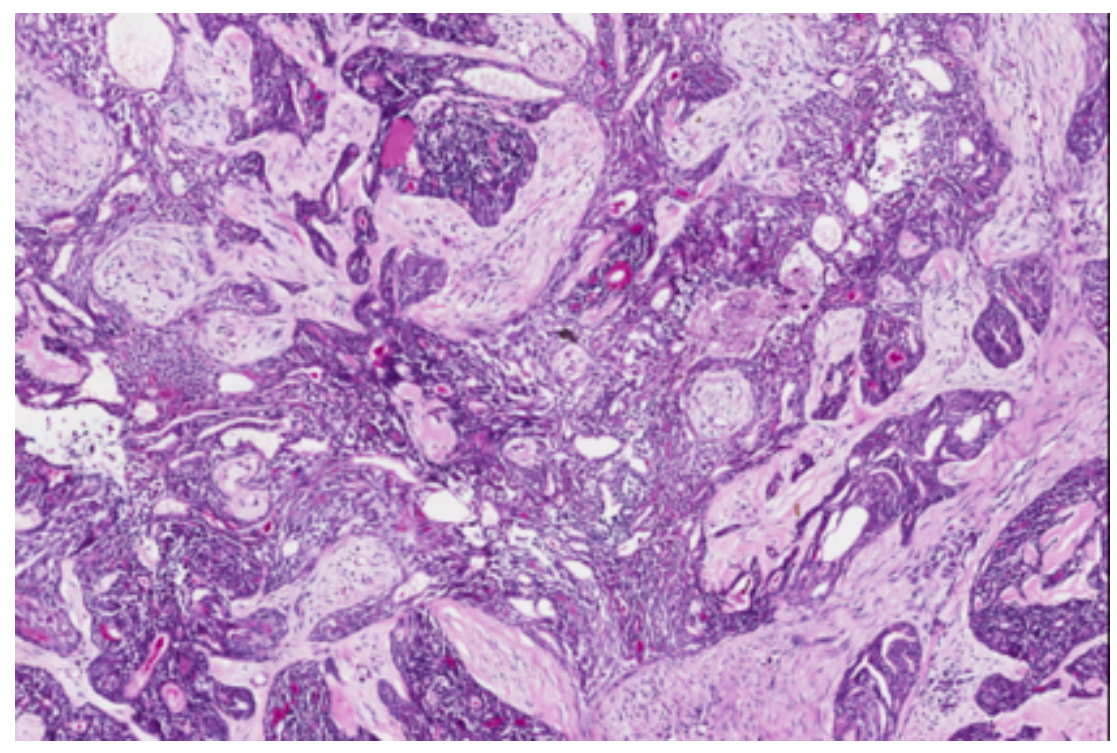

Figure 1

Microscopic picture showing the tumor cells were haphazardly arranged and presented in the shape of ethmoidal foramen (H\&E staining, $x 200)$. 


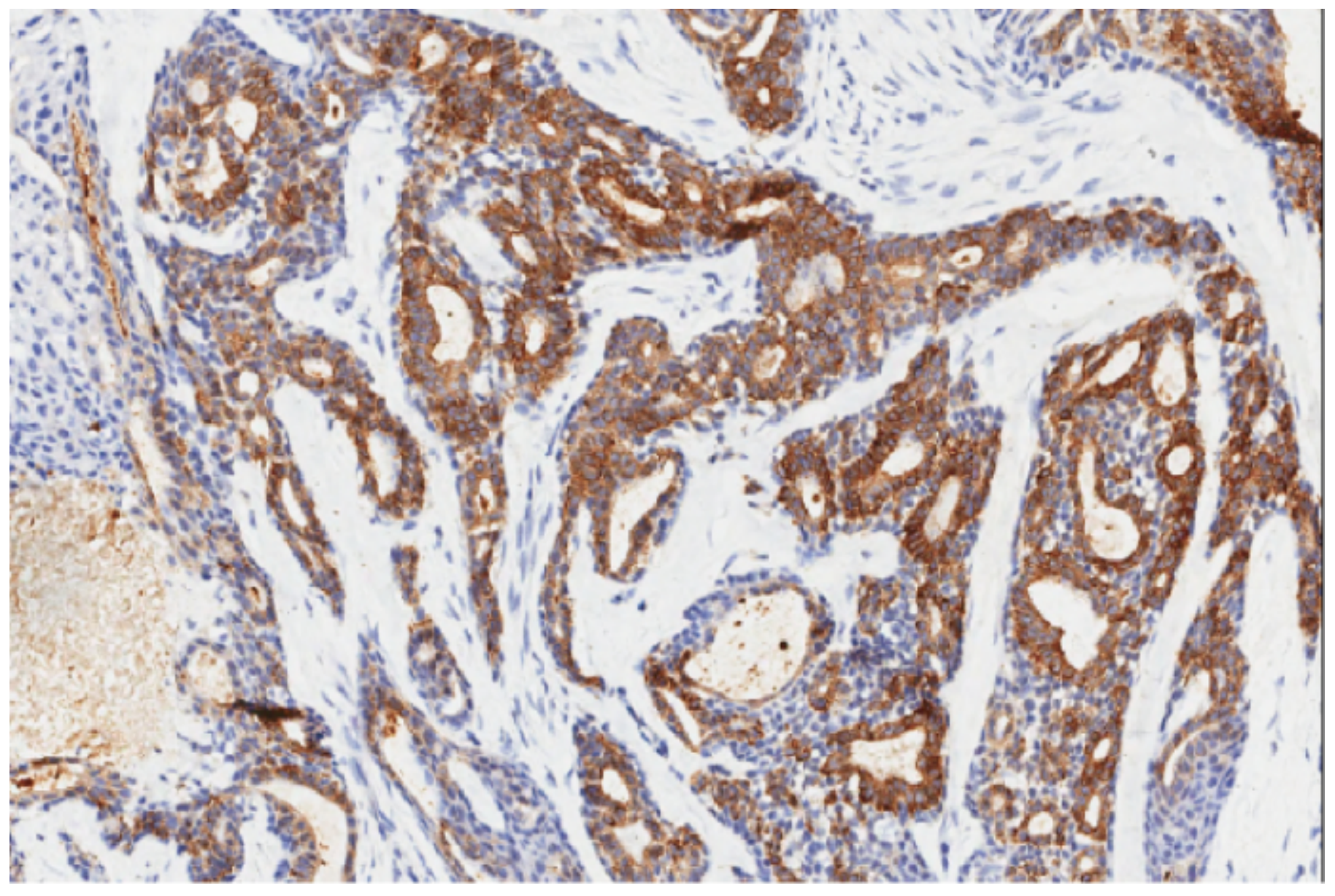

Figure 2

The tumor was positive for CK7 (IHC, x200). 


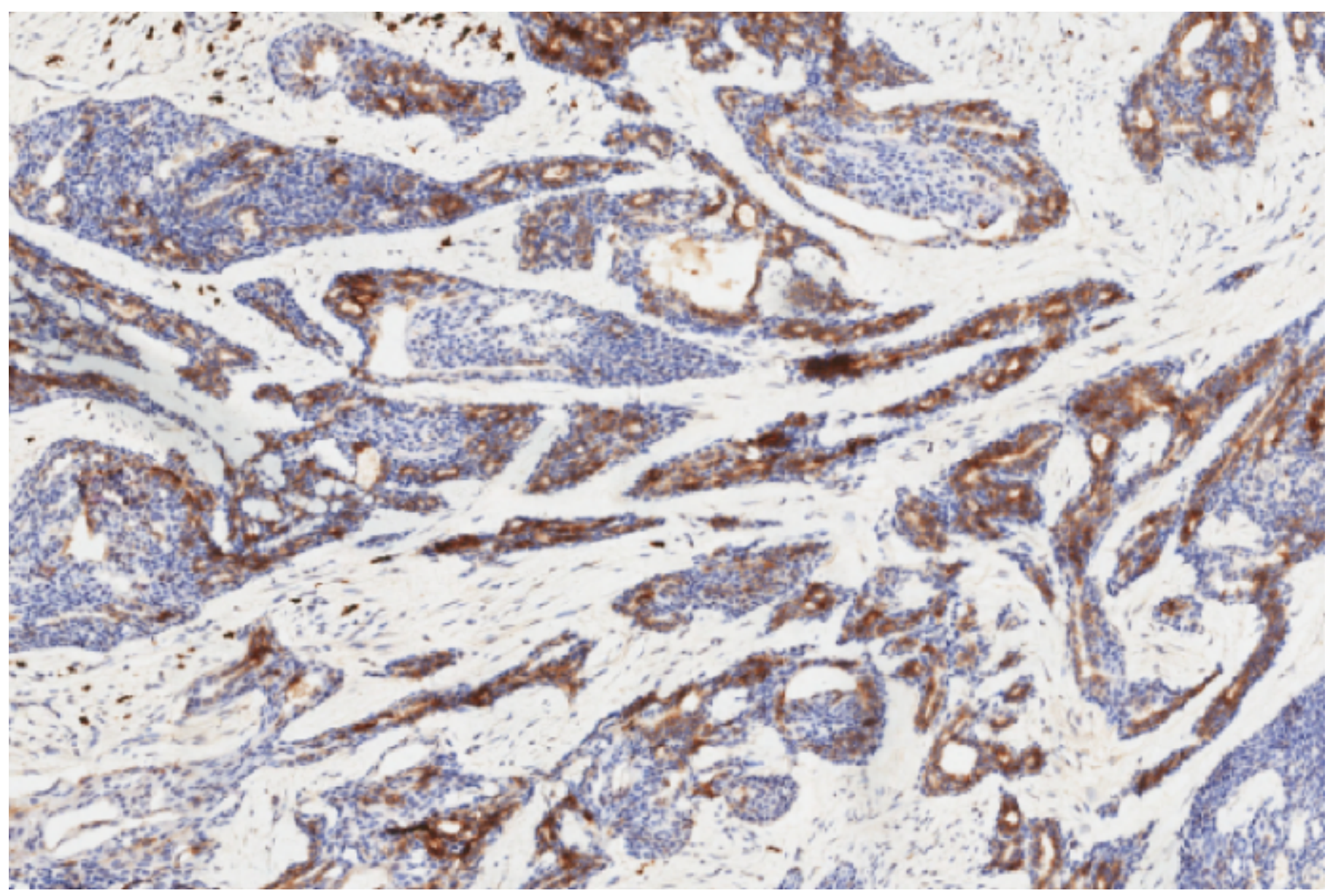

\section{Figure 3}

The tumor was positive for P63 (IHC, x200). 


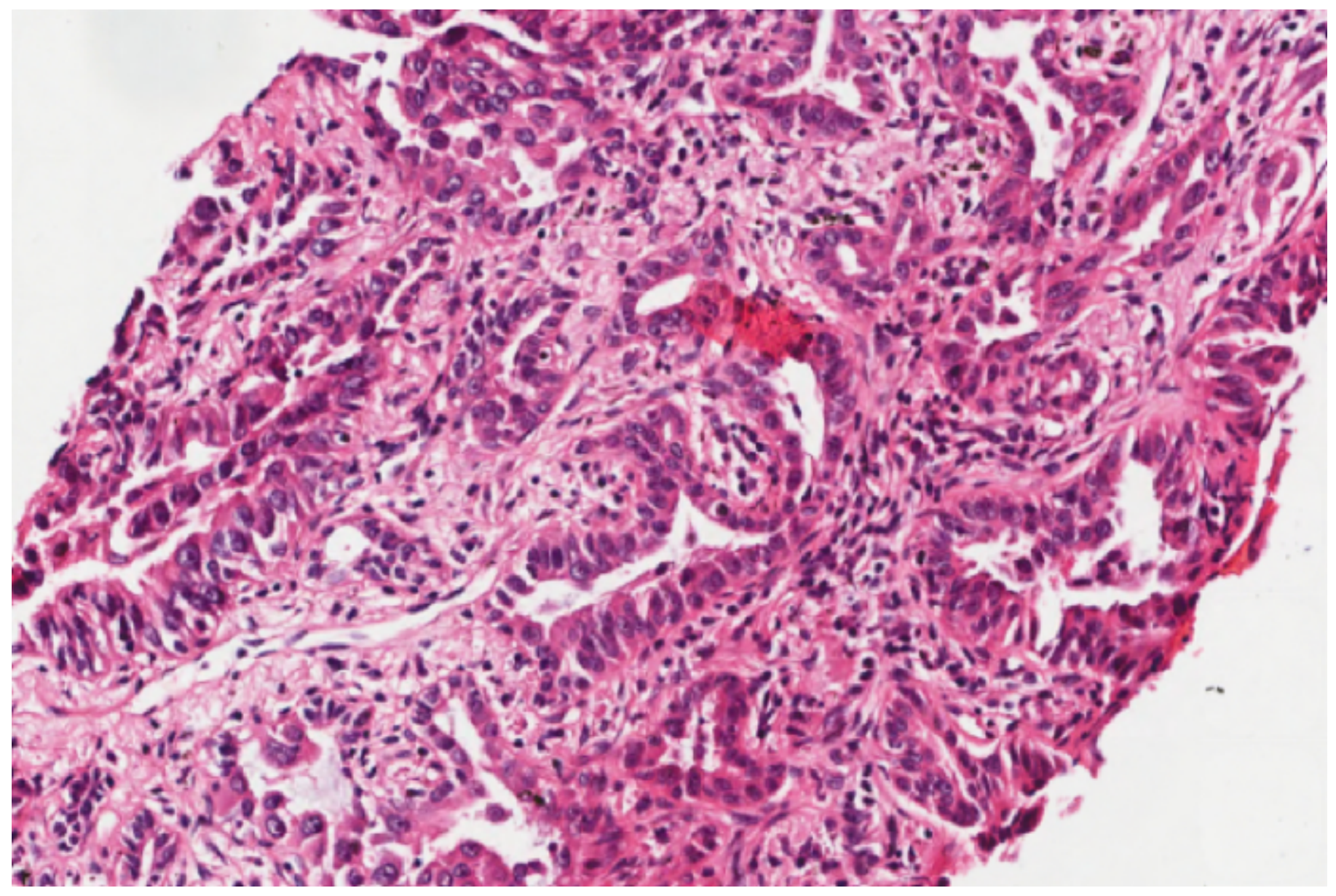

\section{Figure 4}

Microscopic picture showing the tumor cells were arranged in an irregular glandular tube (H\&E staining, x200). 


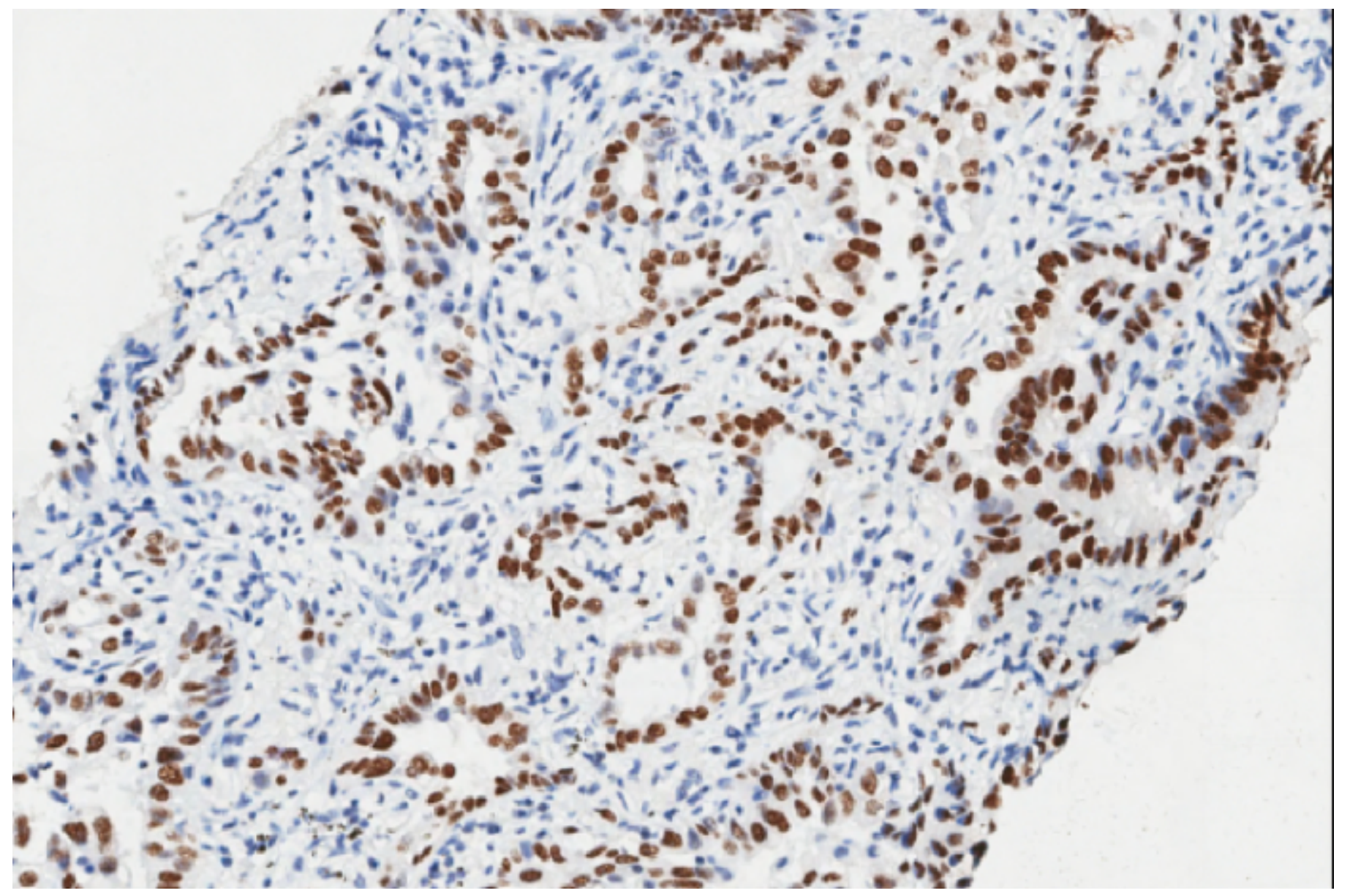

Figure 5

The tumor was positive for TTF-1 (IHC, x200).

\section{Supplementary Files}

This is a list of supplementary files associated with this preprint. Click to download.

- CAREchecklist.pdf 